\title{
28 Research Square \\ Calycosin(CA) Inhibits Proliferation, Migration and Invasion by Suppression of CXCL10 Signaling Pathway in Glioma
}

\section{zhaotao wang}

The Second Affiliated Hospital of guangzhou medical university danmin chen

The Second Affiliated Hospital of guangzhou medical university yongping $\mathrm{Li}$ the second affiliated hospital of guangzhou medical university

renheng Zou

the second affiliated hospital of guangzhou medical university

yunxiang Ji

The Second Affiliated Hospital of guangzhou medical university

Yezhong Wang ( $\sim$ wangyezhong@gzhmu.edu.cn )

Guangzhou Medical University Second Affiliated Hospital https://orcid.org/0000-0002-1664-5765

Primary research

Keywords: Calycosin(CA), CXCL10, Glioblastoma, migration, invasion, proliferation

Posted Date: May 5th, 2021

DOI: https://doi.org/10.21203/rs.3.rs-466485/v1

License: () (1) This work is licensed under a Creative Commons Attribution 4.0 International License. Read Full License 


\section{Abstract}

Background: Glioblastoma is a frequently encountered central nervous system malignancy. Calycosin, a bioactive compound extracted from dried Radix astragali root has exhibited anticancer activity across several different types of cancers, including glioma, but the therapeutic mode of action has yet to be clarified. One ubiquitous inflammatory chemokine implicated in cancer biology is the C-X-C chemokine ligand 10 (CXCL10). The current study seeks to investigate CXCL10 as a likely calycosin target in aiding its inhibitory effects on glioblastoma progression.

Method: The clinical significance of CXCL10 including pathologic grade and survival rate in GBM patients were accessed from TCGA database and our clinical samples. CCK-8 experiments and clone formation assay were performed to detect the cell proliferation. Transwell assay was conducted to examined cell migration and invasion. Western blotting and immunofluorescence staining was used to analyze protein expression levels. Xenograft mouse model was used to evaluate the effect of CA in vivo.

Results: Firstly, we demonstrated that CXCL10 expression is positively associated with glioma and correlated negatively to patient prognosis, supporting our hypothesis that CXCL10 was a potential target for GBM treatment. Additionally, U251 and U87 glioma cell lines were found to demonstrate inhibited invasive, migratory and proliferative abilities upon exposure to calycosin. Calycosin was then found to downregulate CXCL10 expression in a manner that increased with increasing doses, resulting in reduced downstream inflammatory cytokines including NLRP3, NF-KB, IL-1 $\beta$. Moreover, we demonstrated that overexpressing CXCL10 abolished the inhibitory impact of calycosin on cellular invasion, migration and proliferation in glioma cell lines. Meanwhile, CXCL10 knockdown enhanced calycosin effects on GBM cells. We also used in vivo xenograft mice models to determine the impact of calycosin on glioma growth and obtained results similar to our in vitro experiments.

Conclusions: The current investigation underscores the role of CXCL10 as a promising therapeutic target for treating GBM, with calycosin functioning as a future treatment modality.

\section{Introduction}

Glioblastoma (GBM) is an aggressive and unfortunately frequently encountered cerebral malignancy. GBM treatment primarily involves surgery, radiotherapy and chemotherapy. [1, 2]. However, most GBM present as multifocal disease with high degrees of invasiveness. Complete surgical resection is challenging, resulting in abysmally high rates of recurrence and an average survival of a mere 14.6 months after diagnosis[3]. Improved therapeutic methods are necessary to treat GBM.

Calycosin(CA) is a significant bioactive chemical extracted from dried Radix astragali root [4]. As an isoflavonoid and a phytoestrogen, calycosin exerts anti-inflammatory, neuroprotective, and cardiovascular protective properties [5-7]. Recently, it has been reported that calycosin exhibits antitumor activities. For instance, calycosin appears to inhibit breast cancer progression by downregulating Foxp3/VEGF/MMP-9 signaling pathway[8]. In addition, calycosin was demonstrated to induce apoptosis 
via ERßmediated PI3K/Akt signaling pathways in osteosarcoma[9]. Nie et al. verified that calycosin was also effective in inhibiting GBM growth through TGF $\beta$ suppression[10, 11]. However, the underlying mechanisms have yet to be fully outlined.

Chemokines are small secretory immunoregulatory proteins which interact with a subset of seventransmembrane G protein-coupled receptors (GPCRs) in order to regulate cell trafficking. GPCRs also participate in oncogenesis, tumor development and metastatic spread. The C-X-C motif chemokine (CXCL10), also named as the interferon-gamma-induced protein 10 (IP-10), is a $10 \mathrm{kDa}$ polypeptide belonging to the CXC chemokine subfamily. Like most chemokines, CXCL10 exerts multiple

biological functions ranging from mediating immune cell chemotaxis, angiogenesis and the inflammatory response [12-14]. In recent years, some studies have reported that CXCL10 contributed to the progression in various cancers such as colorectal, ovarian, breast and pancreatic cancer [15-18]. Nevertheless, the role of CXCL10 as a tumor suppressor or oncogenic factor in glioma has yet to be confirmed.

Inflammation is a significant instigator in the onset and progression of tumorigenesis[19]. Inflammation accelerates GBM cancer progression while simultaneously promotes treatment-resistance[20]. The NODlike receptor protein-3 (NLRP3) inflammasome is documented to be excessively activated in various cancers including glioma. NLRP3 suppression prolonged the survival of glioma-bearing mice as well as tumor growth. NLRP3 downstream effectors IL-1 $\beta$ and NF-KB were also abundantly found in the GBM tumor microenvironment (TME), and likely contributes to tumor development [21-23]. However, there is limited knowledge regarding the relationship between CXCL10 and NLRP3. Whether or not CA can downregulate CXCL10 to inhibit GBM also reminds unknown.

This series of investigations demonstrates that raised CXCL10 expression was associated with more advanced tumor pathological grade and a poorer prognosis. We also identified CXCL10 to be a potential treatment target of $\mathrm{CA}$ in suppressing GBM, which likely works by downregulating downstream molecules such as NLRP3, NF-KB and IL-1 $\beta$ both in vivo and in vitro. These findings suggest that CXCL10 was a novel biomarker in glioblastoma and CA may be a potential treatment agent in GBM.

\section{Materials And Methods}

\section{Reagents}

Calycosin was purchased from Tianjin Wanxiang Hengyuan Science and Technology Ltd (Tianjin, China). Dulbecco's Modified Eagle Medium (DMEM) and Fetal Bovine Serum (FBS) were purchased from Gibco ${ }^{\mathrm{TM}}$. Antibody against CXCL10, NLRP3 and IL-1 $\beta$ were purchased from Cell Signaling Technology. Antibodies against NF-KB were purchased from Wuhan Servicebio Technology Co. Ltd. (Wuhan, China)

\section{Cell Culture}


The human GBM cell lines U87 and U251 were purchased from iCell Bioscience Inc (Shangha, China). These cell lines were cultured in Dulbecco's Modified Eagle Medium (DMEM) supplemented with 10\% fetal bovine serum (FBS) and incubated at $37^{\circ} \mathrm{C}$ in a humidified atmosphere in $5 \% \mathrm{CO}_{2}$.

\section{Cell Viability Assay}

4000 cells/well were planted in a 96-well plate and treated with different doses of calycosin for $24 \mathrm{~h}$. Then, $10 \mu$ l of the CCK-8 solution (Beyotime, Shanghai, China) was added to each well and incubated for $1 \mathrm{~h}$ at $37^{\circ} \mathrm{C}$. Then, the reaction mixture was measured by Multimode Reader.

\section{Colony formation assay}

500 cells/well were seeded in $60 \mathrm{~mm}$ dishes and treated with different doses of calycosin for 2 weeks. Colonies were counted after fixing by methanol and staining with Crystal Violet Staining Solution (Beyotime, Shanghai, China).

\section{Cell Invasion Assay}

The transwell system for assay of cell migration and invasion was purchased from Corning (Corning, USA). $2 \times 10^{5}$ cell/well ( $200 \mu$ I DMEM supplemented with $1 \%$ FBS) were seeded in the upper chamber (8 $\mu \mathrm{m}$ Pore Polycarbonate Membrane) coated with o $100 \mu \mathrm{l}$ matrigel (BD Biosciences, CA, USA). The lower chamber was filled without or with $600 \mu$ DMEM supplemented with $20 \%$ FBS and indicated different concentrations of calycosin. After $24 \mathrm{~h}$, the cells in the lower chamber were fixed by methanol and stained with Crystal Violet Staining Solution (Beyotime, Shanghai, China). Five independent fields in each well were photographed at 100×magnification, after which the cells in each field were counted.

\section{Western Blotting}

Western blotting was performed using glioblastoma cell lysates, clinical normal or tumor tissues homogenates and xenograft glioblastoma tissue homogenates. Protein was extracted using PRO-PREP ${ }^{\text {TM }}$ Protein Extraction Solution (Cell/Tissue) (iNtRON Biotechnology, Korea) according to manufacturer's instructions. Equal amounts of total protein were separated on $10-12 \%$ sodium dodecyl sulfatepolyacrylamide gel electrophoresis (SDS-PAGE), transferred to polyvinylidene difluoride membranes (Merck, KGaA, Darmstadt, Germany). The membranes were blocked with $5 \%$ BSA at room temperature for $1 \mathrm{~h}$, and then incubated with specific primary antibodies overnight at $4{ }^{\circ} \mathrm{C}$. The appropriate secondary antibodies conjugated with HRP were incubated for $1 \mathrm{~h}$ at room temperature, signal was obtained using Immobilon Western HRP Substrate (Merck, KGaA, Darmstadt, Germany).

\section{Transfection}

To overexpression CXCL10, glioblastoma cell lines were transfected with lentiviral carrying CXCL10-flag or its vector (OBiO Technology Corp., Ltd, Shanghai, China). To knockdown of CXCL10, glioblastoma cell lines were transfected with CXCL10 siRNA or empty vector (GenePharma, Shanghai, China) using jetPRIME ${ }^{\circledR}$ Versatile DNA/siRNA transfection reagent (Polyplus Transfection, France) following the 
manufactory protocol. CXCL10 siRNA: sense 5》-CCUUAUCUUUUCUGACUCUATT-3》; antisense 3囚UAGAGUCAGAAAGAUAAGGTT-5】.

\section{U87 Xenograft Mouse Model Paeoniflorin Treatment}

Female BALB/c nude mice were obtained from Guangdong Medical Laboratory Animal Center (Guangdong, China). Mice were aged 6-8 weeks and kept under a standard protocol approved by the Laboratory Animal Center of the Second Affiliated Hospital of Guangzhou Medical University. All procedures performed in studies involving animals were in accord with the ethical standards of the Ethics Committee. Each mouse was injected subcutaneously with cultured U87 cells $\left(5 \times 10^{6}\right.$ cells/mouse) into the dorsum. The tumor size was measured in two orthogonal directions using calipers, and the tumor volume $\left(\mathrm{mm}^{3}\right)$ was calculated using the equation: $1 / 2 \times$ length $\times$ width ${ }^{2}$. When the tumors grew to about $150 \mathrm{~mm}^{3}$, the tumor-bearing mice were distributed into two groups ( $\mathrm{n}=5 \mathrm{each}$ ) and orally fed with calycosin $(10 \mathrm{mg} / \mathrm{kg} /$ day) or vehicle (equivalent amount of PBS) Tumor sizes and body weights were measured once every 5 days. At the end of these experiments, the mice were sacrificed and the tumors were resected and homogenized for western blotting.

\section{Statistical analysis}

All data are composites of three repeated experiments and were analyzed using the SPSS 20.0 software. An independent T-test was used to perform simple comparisons between 2 groups and multiple comparisons between the groups were evaluated using one-way analysis of variance, followed by post hoc analyses, which were carried out using Dunnett's T3 test or Turkey test. $\mathrm{P}<0.05$ was interpreted as achieving statistical significance.

\section{Results}

\section{CXCL10 is upregulated in glioma tissues and contributes to poor prognosis}

CXCL10 mRNA expressions were first quantified in glioma and normal brain tissues based on publicly accessible data from The Cancer Genome Atlas (TCGA) and GTEx database as well as in 24 pairs of glioma tissue samples and matched adjacent normal brain tissue samples. CXCL10 expression levels were significantly raised in glioma tissues in contrast to their adjacent healthy tissues (Fig. 1A, D-E). CXCL10 mRNA expression were significantly increased based on more advanced WHO GBM grade (Fig. 1B, F-G). Furthermore, as shown in Fig. 1C and H, patients with elevated CXCL10 expression had markedly decreased overall survival (OS) in contrast to patients with lower CXCL10 expressions. These results suggest that high CXCL10 expression correlates positively to poorer prognosis in GBM patients.

\section{CA inhibited cells growth and downregulated CXCL10 signaling in GBM cells}


We evaluated the impact of CA on GBM, CCK-8 experiments was performed. CA treatment markedly decreased the degree of cell proliferation in U87 and U251 GBM cells in a time- and dose-dependent manner. An increase in CA concentration from 100 to $400 \mu \mathrm{M}$ resulted in reduced cell viability from 90$40 \%$ in both cell lines (Fig. 2A-B). CA effects on GBM cell growth were evaluated using colony formation assays. Likewise, the number of viable cell colonies were reduced from 50 to 5 in a dose-dependent manner. In conclusion, CA appears to have a suppressive effect on in vitro GBM cell growth (Fig. 2C-D).

CA effects on the migratory and invasive abilities of cells were also studied. In contrast to the untreated groups, the CA treated groups exhibited reduced cell invasiveness and cell migration in a dose-dependent manner (Fig. 2E-F). These results demonstrate that CA significantly inhibits GBM cell migration and invasion.

As mentioned above, CXCL10 is upregulated in GBM tissues and contributes to tumor progression and a poorer patient prognosis. Suppressing CXCL10 appears to be a promising therapeutic modality for GBM. GBM cell exposed to CA demonstrated downregulated expressions of CXCL10 and its downstream molecules such as NLRP3, NF-KB and IL-1 $\beta$. As shown in Fig. 2I, CA reduced CXCL10, NLRP3, NF-KB and IL-1 $\beta$ expressions in a dose-dependent manner.

\section{Overexpression of CXCL10 reduced the effects of calycosin in GBM cells}

To further characterize whether CA inhibited GBM cell growth by downregulating CXCL10, U87 and U251 cells with a lentiviral vector carrying CXCL10 CDNA. The results showed that overexpressed CXCL10 enhanced U87 and U251 cells invasive, migratory and proliferative abilities. Furthermore, overexpressing CXCL10 reduced the suppressive properties of CA on U87 and U251 cells (Fig. 3A-H). We also found that overexpressing CXCL10 promoted NLRP3, NF-KB, IL-1 $\beta$ expression (Fig. I).

\section{Downregulation of CXCL10 enhances the effects of calycosin in GBM cells}

To clarify the tumorigenic role of CXCL10, CXCL10 expression was knocked down in CA-treated GBM tumors using specific siRNA. CCK-8 and colony formation experiments showed that knockdown of CXCL10 enhanced CA-induced suppression of cells proliferation (Fig. 4A-D). Both CXCL10 siRNA or CA strongly inhibited cell migratory and invasive capabilities (Fig. 4E-H). These results indicated that combination treatments demonstrated a significantly greater effect compared to any agent given in isolation. Our results also demonstrated that CA treatment decreased NLRP3, NF-KB and IL-1 $\beta$ expressions while additional CXCL10 suppression resulted in even lower inflammatory marker expressions (Fig. 4I). Taken together, these experiments highlight the that the anticancer CA property was at least partly mediated through downregulating CXCL10.

\section{CA inhibits GBM Growth in a U87 Xenograft Mouse Model}


A U87 xenograft mouse model was used to evaluate the inhibitory effects of CA on the progression of GBM. As shown in Fig. 5A-C, mice treated with CA had much smaller tumor volumes in contrast to the control group. Body weights of mice from both groups were similar after 20 days of treatment with either CA or vehicle (Fig. 5D). Furthermore, the U87 xenograft mouse models treated with CA had lower CXCL10, NLRP3, NF-KB and IL-1 $\beta$ tumor expressions. CA appeared to remarkably suppress the CXCL10 pathway

(Fig. 5E). Our results from the in vivo studies are similar to those gained in our in vitro experiments, further highlighting the central role of CA in inhibiting GBM progression.

\section{Discussion}

In present study, we proved that overexpressing CXCL10 promoted GBM progression. Moreover, we demonstrated that CA was able to inhibit growth, migration and invasion in GBM via downregulation of CXCL10 pathway. We also verified that CA exerts anti-tumor effect in a GBM xenograft mouse model. Similar findings were seen in both our in vitro and in vivo experiments.

Current treatment modalities available to treat GBM comprises primarily of surgical resection along with supporting chemoradiotherapy. Curative resection is challenging given the multifocal and aggressive nature of disease [1]. This study identifies calycosin, a natural bioactive chemical extracted from dried Radix astragali root to exert anti-tumor properties through suppressing CXCL10 expression in GBM.

Calycosin has shown anti-tumor effects across several different types of malignancies, including glioma [8-10]. A previous study by our group found that CA exerted anti-GBM effects by downregulating TGF $\beta$ and c-Met $[10,11]$. Furthermore, Ni Q et al reported that the combination of CA and temozolomide enhanced anti-tumor effects in GBM [24]. However, the underlying molecular target of CA in GBM remains to be completely investigated. In our study, we verified that CA inhibited GBM growth both in vitro and in vivo through inhibition of the CXCL10 signaling pathway, indicating that the CXCL10 pathway may be critical in treating GBM.

CXCL10 is involved in the regulation of multiple inflammatory signaling pathways, which are closely linked with tumor development and occurrence. However, the role of CXCL10 in GBM progression remains controversial. Maru SV et al reported that overexpression CXCL10 significantly impacts glioma cell proliferation [25]. A recent immunohistochemical study found that CXCL10 was overexpressed in GBM [26]. Kenji Shono et al revealed that CXCL10 exerts anti-tumor impact in a mouse model of malignant glioma [27]. CXCL10 appears to be critical regulator of GBM growth and progression. Suppressing CXCL10 may potentially impart positive effects in treating GBM. However, there are contradictory studies which have found that CXCL10 upregulation inhibits GBM progression $[28,29]$. In current study, we demonstrated that CXCL10 was obviously elevated in GBM and is negatively associated with patient prognosis, based on data gained from publicly accessible databases and actual patient tissue samples. We therefore hypothesize that CXCL10 functions as an oncogene in GBM. Furthermore, we confirmed that downregulating CXCL10 suppressed the inflammatory signaling pathway which further inhibited GBM progression. 
Many agents exert in vitro anti-GBM effects but fail to yield similar results in GBM orthotopic xenograft mouse models due to the existence of blood-brain barrier (BBB). Previous studies on cerebral ischemic mouse models and brain reperfusion injury models found that CA exerted remarkable therapeutic effects in brain disease and showed a good safety profile [5]. This study finds that CA inhibited GBM growth by downregulating the CXCL10 signaling pathway in a GBM mouse model. CA also decreased tumor volume without significant changes in body weight, which further verifies the in vivo safety and efficacy profiles of CA.

Overall, we demonstrated that CXCL10 functions as an oncogene and holds to potential as a potential therapeutic target of CA in glioma.

\section{Declarations}

\section{Ethics approval and consent to participate}

Not applicable.

\section{Consent for publication}

Not applicable.

\section{Availability of data and materials}

The datasets used and/or analyzed during the current study are available from the corresponding author on reasonable request.

\section{Competing interests}

The authors declare that they have no competing interests.

\section{Funding}

This work was supported by the National Natural Science Foundation of China (81901117) and Natural Science Foundation of Guangdong Province (2019A1515010926).

\section{Authors' contributions}

Yunxiang Ji and Yezhong Wang designed the study, Zhaotao Wang and Danmin Chen performed the experiments and prepared the figures; all authors contributed to drafting the manuscript, read and approved the final manuscript.

\section{Acknowledgements}

Not applicable. 


\section{References}

1. Batash R, Asna N, Schaffer P, et al. Glioblastoma multiforme, diagnosis and treatment; recent literature review. Curr Med Chem. 2017;24:3002-9.

2. Anjum K, Shagufta Bl, Abbas SQ, et al. Current status and future therapeutic perspectives of glioblastoma multiforme (gbm) therapy: A review. Biomed Pharmacother. 2017;92:681-9.

3. Taylor OG, Brzozowski JS, Skelding KA. Glioblastoma multiforme: An overview of emerging therapeutic targets. Frontiers in oncology. 2019;9:963.

4. Gao J, Liu ZJ, Chen T, et al. Pharmaceutical properties of calycosin, the major bioactive isoflavonoid in the dry root extract of radix astragali. Pharmaceutical biology. 2014;52:1217-22.

5. Guo C, Tong L, Xi M, et al. Neuroprotective effect of calycosin on cerebral ischemia and reperfusion injury in rats. J Ethnopharmacol. 2012;144:768-74.

6. Dong L, Yin L, Chen R, et al. Anti-inflammatory effect of calycosin glycoside on lipopolysaccharideinduced inflammatory responses in raw 264.7 cells. Gene. 2018;675:94-101.

7. Pan Q, Ban Y, Khan S. Antioxidant activity of calycosin against a-synuclein amyloid fibrils-induced oxidative stress in neural-like cells as a model of preventive care studies in parkinson's disease. International Journal of Biological Macromolecules, 2021.

8. Li S, Wang Y, Feng C, et al. Calycosin inhibits the migration and invasion of human breast cancer cells by down-regulation of foxp3 expression. Cell Physiol Biochem. 2017;44:1775-84.

9. Tian W, Wang ZW, Yuan BM, et al. Calycosin induces apoptosis in osteosarcoma cell line via er $\beta-$ mediated pi3k/akt signaling pathways. Mol Med Rep. 2020;21:2349-56.

10. Nie X-h, Ou-yang J, Xing Y, et al. Calycosin inhibits migration and invasion through modulation of transforming growth factor beta-mediated mesenchymal properties in u87 and u251 cells. Drug Des Devel Ther. 2016;10:767.

11. Nie X, Zhou Y, Li X, et al. Calycosin down-regulates c-met to suppress development of glioblastomas. Journal of biosciences. 2019;44:1-8.

12. Lee EY, Lee Z-H, Song YW. Cxcl10 and autoimmune diseases. Autoimmun rev. 2009;8:379-83.

13. Yates-Binder CC, Rodgers $M$, Jaynes J, et al. An ip-10 (cxcl10)-derived peptide inhibits angiogenesis. PloS one. 2012;7:e40812.

14. Zhao Q, Kim T, Pang J, et al. A novel function of cxcl10 in mediating monocyte production of proinflammatory cytokines. J Leukoc Biol. 2017;102:1271-80.

15. Hirth M, Gandla J, Höper C, et al. Cxcl10 and ccl21 promote migration of pancreatic cancer cells toward sensory neurons and neural remodeling in tumors in mice, associated with pain in patients. Gastroenterology. 2020;159:665-81. e613.

16. Goldberg-Bittman L, Neumark E, Sagi-Assif O, et al. The expression of the chemokine receptor cxcr3 and its ligand, cxcl10, in human breast adenocarcinoma cell lines. Immunology letters. 2004;92:1718. 
17. Bronger $\mathrm{H}$, Singer J, Windmüller $\mathrm{C}$, et al. Cxcl9 and cxcl10 predict survival and are regulated by cyclooxygenase inhibition in advanced serous ovarian cancer. British journal of cancer. 2016;115:553-63.

18. Chen J, Chen Q-L, Wang W-H, et al. Prognostic and predictive values of cxcl10 in colorectal cancer. Clin Transl Oncol. 2020;22:1548-64.

19. Karki R, Kanneganti T-D. Diverging inflammasome signals in tumorigenesis and potential targeting. Nat Rev Cancer. 2019;19:197-214.

20. Cho A, McKelvey KJ, Lee A, et al. The intertwined fates of inflammation and coagulation in glioma. Mamm Genome. 2018;29:806-16.

21. Shang $S$, Wang $L$, Zhang $Y$, et al. The beta-hydroxybutyrate suppresses the migration of glioma cells by inhibition of nlrp3 inflammasome. Cell Mol Neurobiol. 2018;38:1479-89.

22. Yin X, Zhang Q, Chen Z, et al. Nlrp3 in human glioma is correlated with increased who grade, and regulates cellular proliferation, apoptosis and metastasis via epithelial-mesenchymal transition and the pten/akt signaling pathway. Int J Oncol. 2018;53:973-86.

23. Sharma B, Kanneganti T. Nlrp3 inflammasome in cancer and metabolic diseases. Nature immunology, 2021.

24. Ni Q, Fan Y, Zhang X, et al. In vitro and in vivo study on glioma treatment enhancement by combining temozolomide with calycosin and formononetin. J Ethnopharmacol. 2019;242:111699.

25. Maru S, Holloway K, Flynn G, et al. Chemokine production and chemokine receptor expression by human glioma cells: Role of cxcl10 in tumour cell proliferation. J Neuroimmunol. 2008;199:35-45.

26. Sharma I, Siraj F, Sharma K, et al. Immunohistochemical expression of chemokine receptor cxcr3 and its ligand cxcl10 in low-grade astrocytomas and glioblastoma multiforme: A tissue microarray-based comparison. J Cancer Res Ther. 2016;12:793-7.

27. Shono K, Yamaguchi I, Mizobuchi Y, et al. Downregulation of the ccl2/ccr2 and cxcl10/cxcr3 axes contributes to antitumor effects in a mouse model of malignant glioma. Scientific reports. 2020;10:1-10.

28. Wang $P$, Peng $X$, Zhang J, et al. Lncrna-135528 inhibits tumor progression by up-regulating cxcl10 through the jak/stat pathway. Apoptosis: an international journal on programmed cell death. 2018;23:651-66.

29. Takano S, Ishikawa E, Matsuda M, et al. Interferon- $\beta$ inhibits glioma angiogenesis through downregulation of vascular endothelial growth factor and upregulation of interferon inducible protein 10. Int J Oncol. 2014;45:1837-46.

\section{Figures}



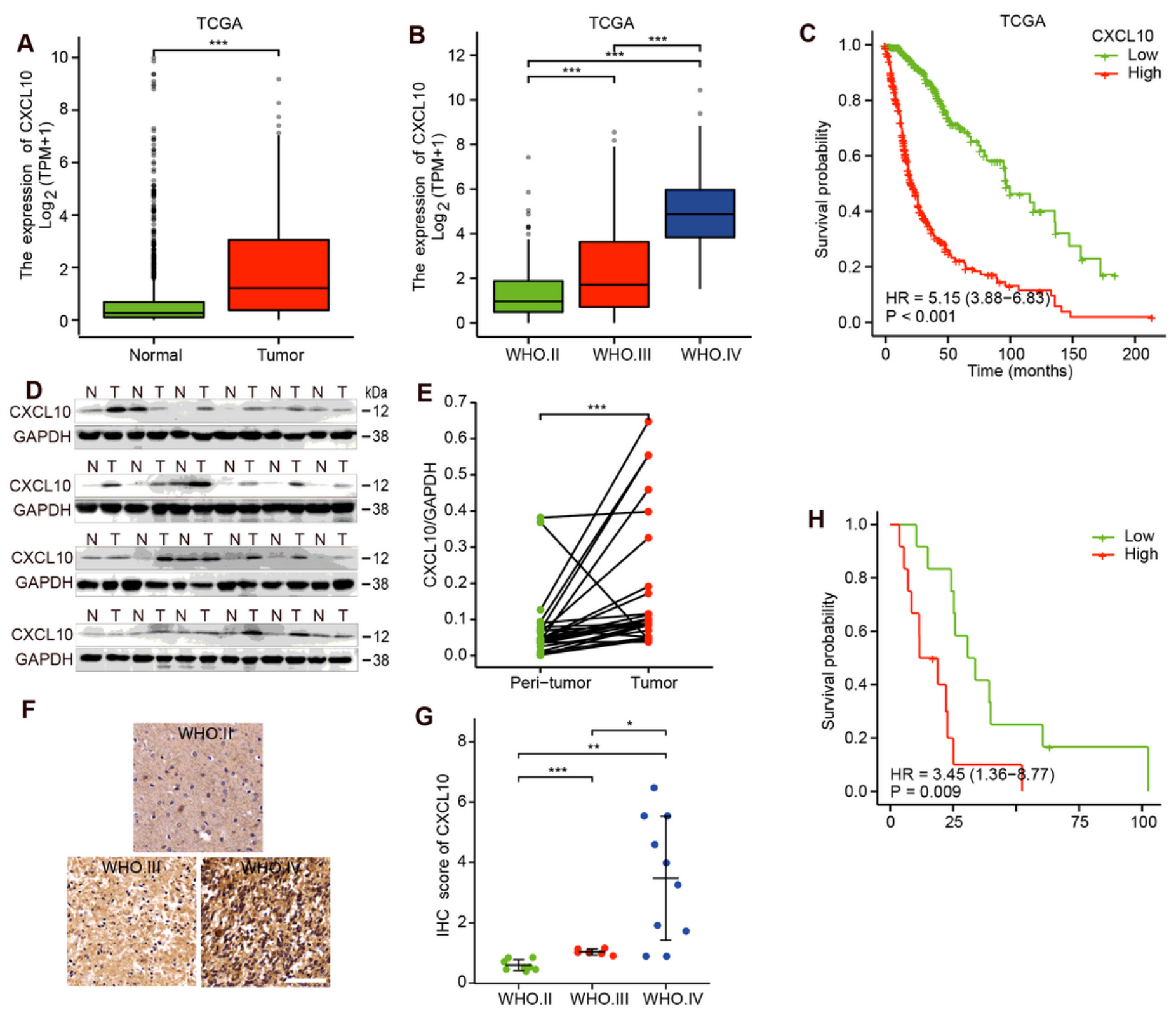

Figure 1

GBM tissues have upregulated CXCL10 levels which is positively associated with tumor progression and poor prognosis. CXCL10 expression in GBM tumor and normal brain tissues were statistically analyzed in the TCGA database (A) and 24 paired tumor and peritumor tissues from GBM patients (D-E). The CXCL10 expression across different grades of GBM were statistically analyzed in the TCGA database $(E)$ and 24 gliomas (F-G). Survival curves for overall survival (OS) were stratified based on CXCL10 expression in GBM tissues derived from TCGA (C) and 24 gliomas $(\mathrm{H})$. 
A

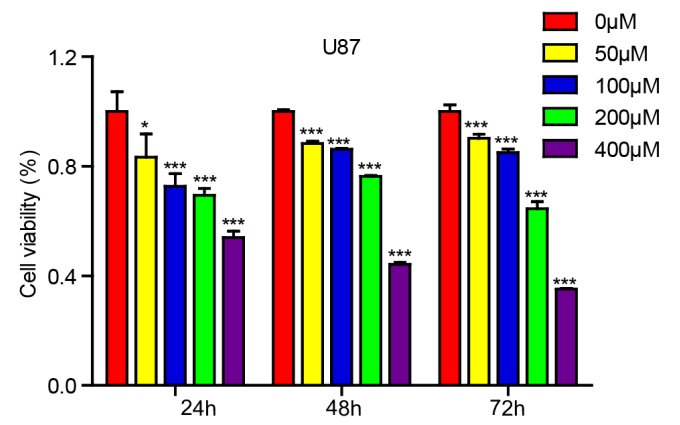

C

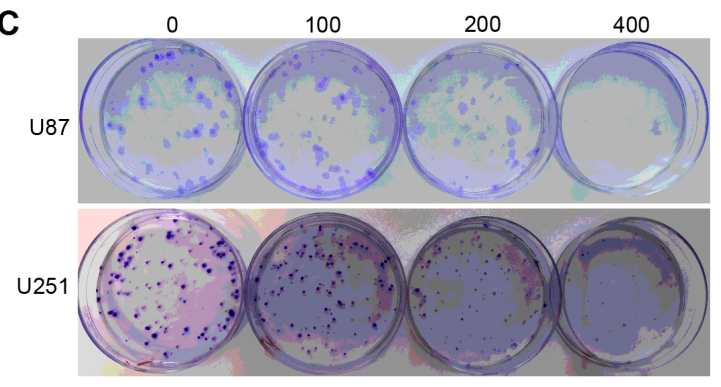

E

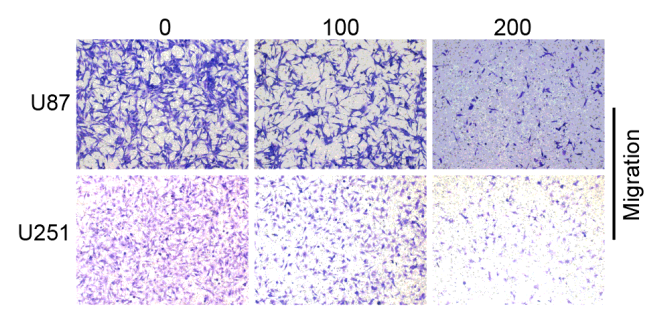

G

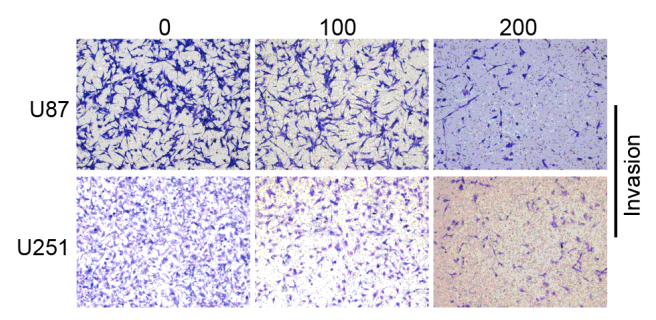

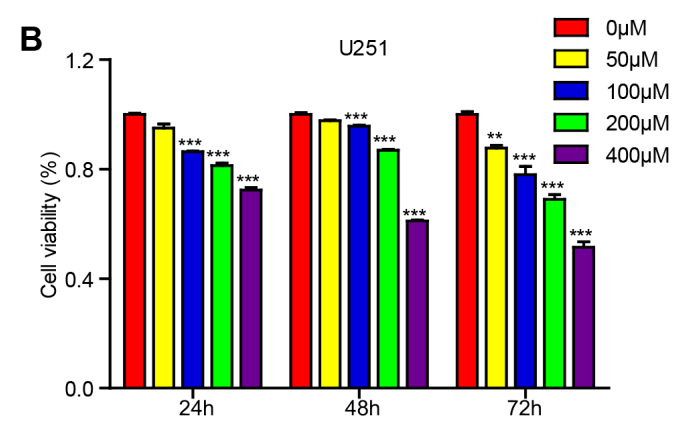

D
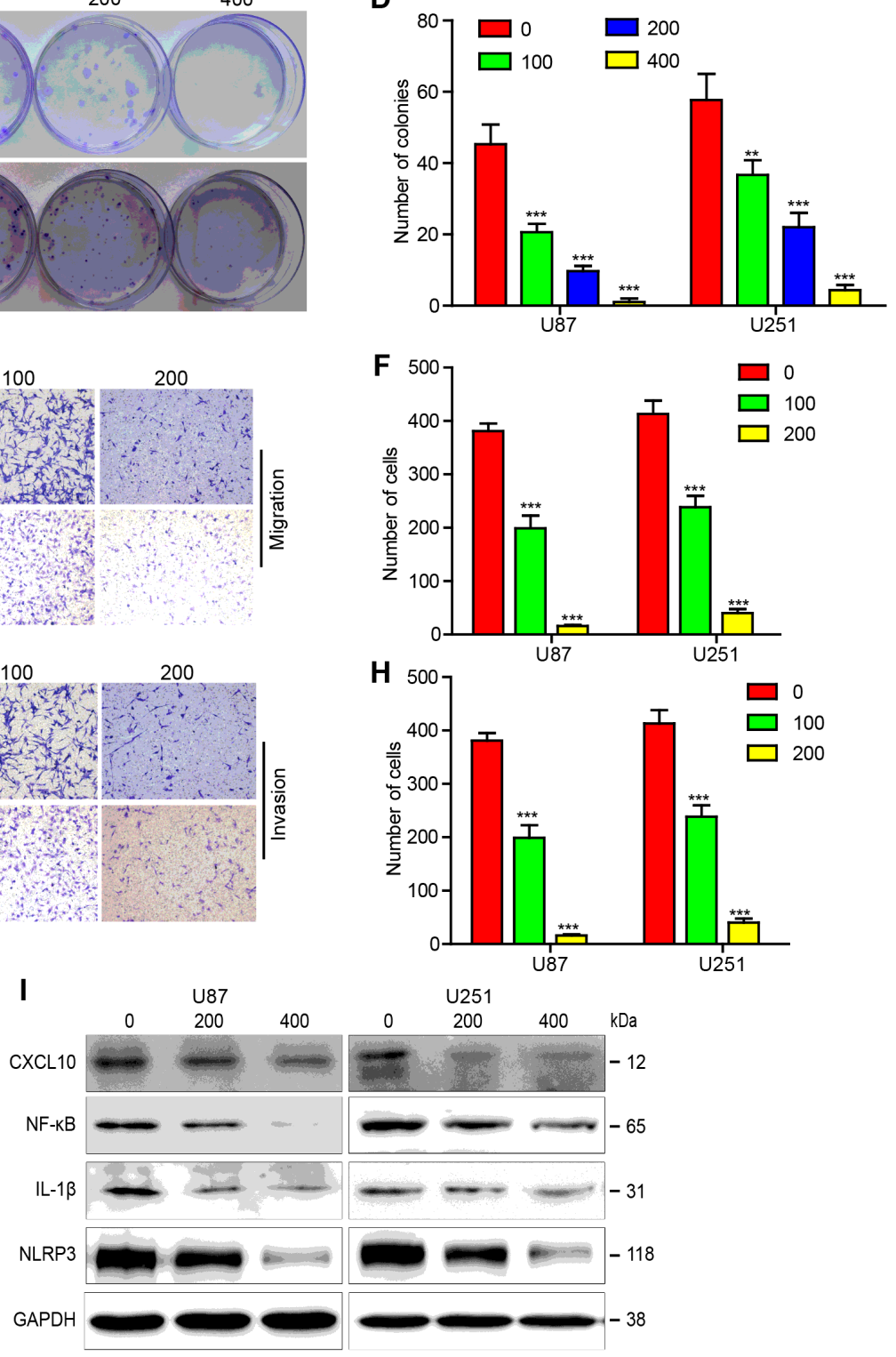

\section{Figure 2}

Calycosin suppressed GBM progression and downregulated the CXCL10 signalling pathway in U87 and U251 cells. (A-B) CCK8 analysis was performed in U87 and U251 cells using different concentrations of CA. (C-D) Cell colony formation of GBM cells treated with different doses of CA (E-H) U87 and U251 cells were exposed for 24 hours to the predetermined dose of CA before transwell assay analysis to determine the degree of cell invasion and migration (I) U87 and U251 cells were exposed to 24 hours with various 
doses of CA prior to Western blot analysis to determine the relative protein levels of components of the CXCL10 pathway. ${ }^{*} P<0.05,{ }^{*} P<<0.01,{ }^{*} *{ }^{*} P<0.001$, compared with control $(0 \mu M)$.

A
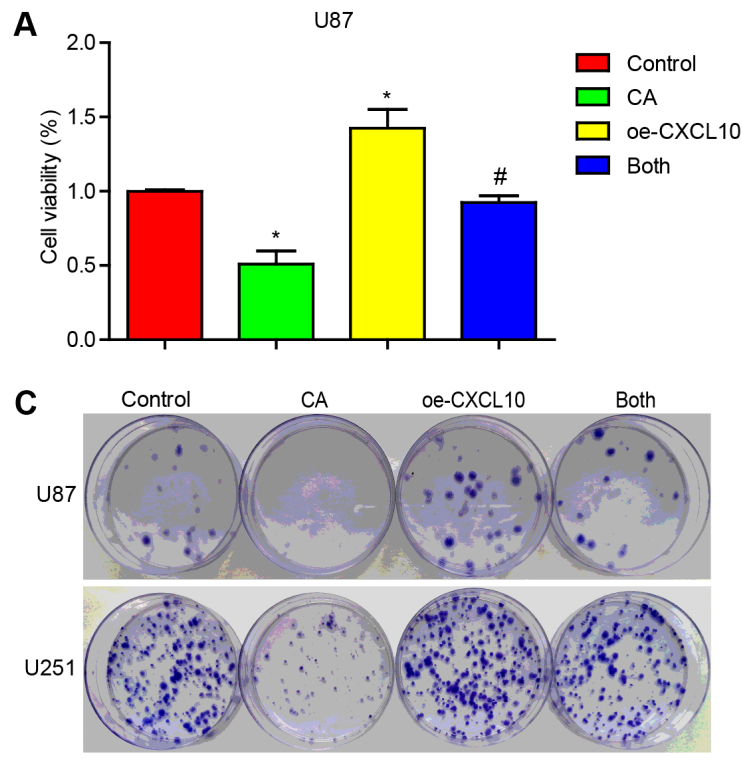

E
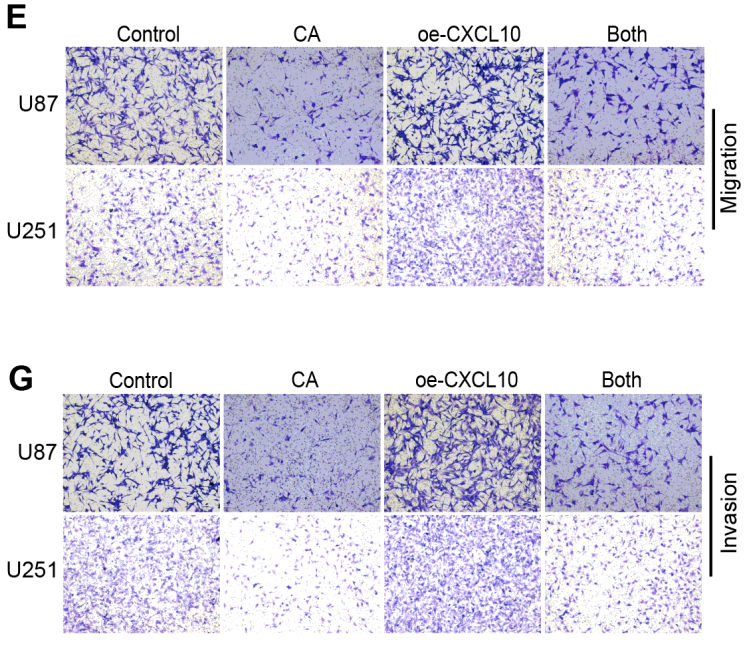

B
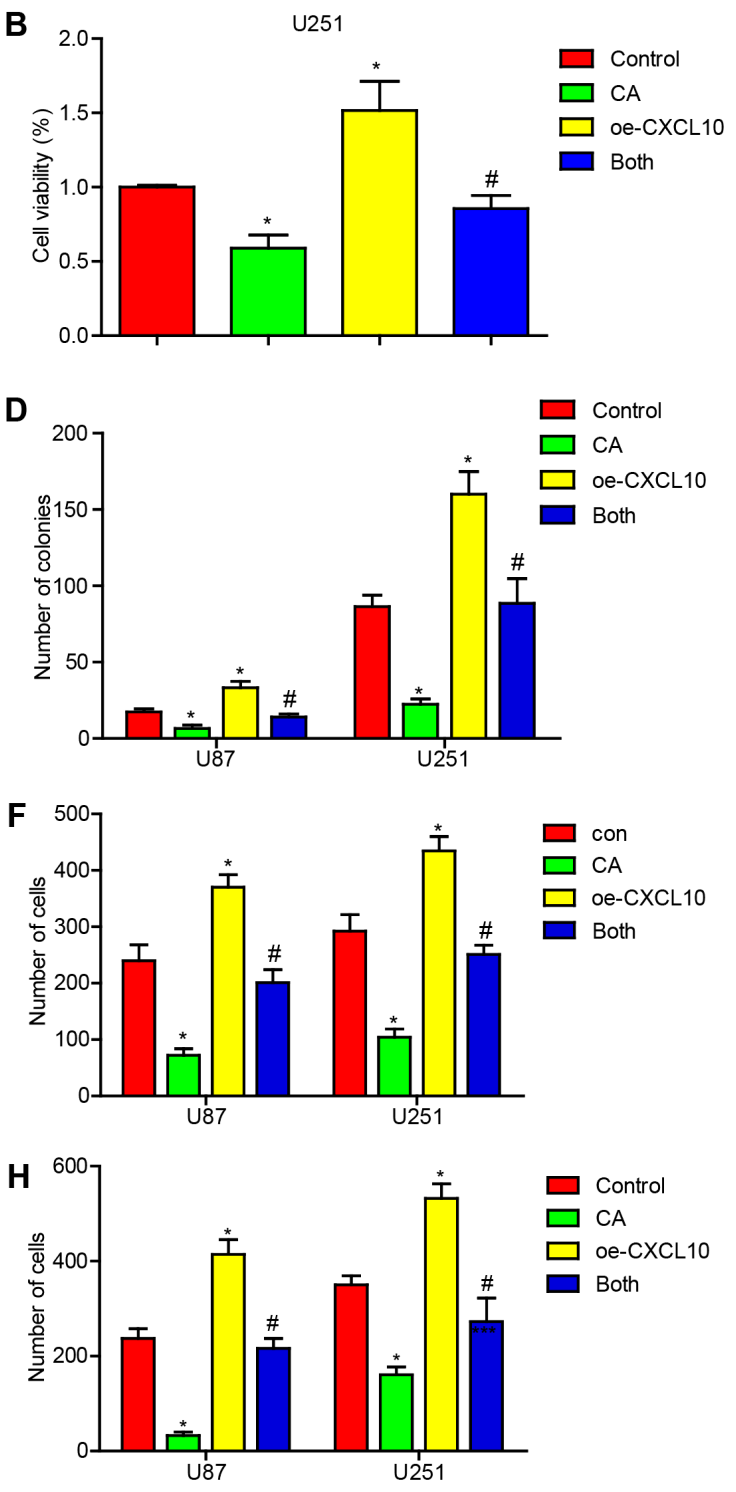

I

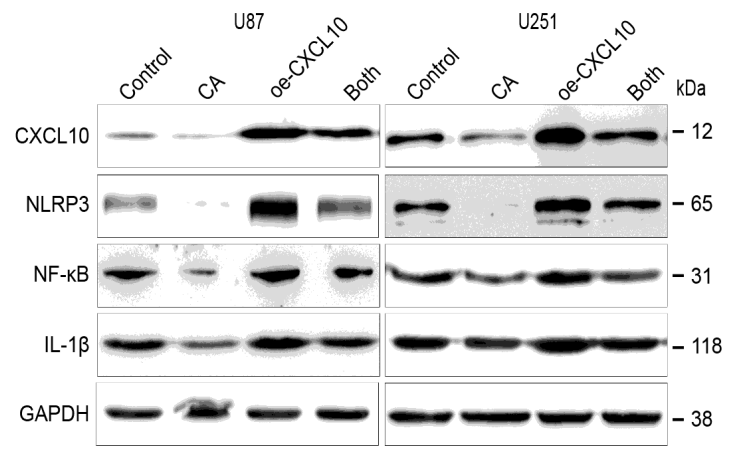

Figure 3

CXCL10 overexpression inhibits calycosin effects on the invasive, migratory and proliferative abilities of GBM cells. (A-D) Effects of CXCL10 overexpression on the proliferative abilities of CA-treated U87 and U251 cells. (E-H) Effects of CXCL10 overexpression on the migratory and invasive abilities of CA-treated 
U87 and U251 cells. (I) Overexpressing CXCL10 rescues CA-induced CXCL10, NLRP3, NF-KB and IL-1 $\beta$ downregulation. Control: GFP lentivirus transfection; CA: GFP lentivirus transfection $+200 \mu \mathrm{M}$ calycosin;

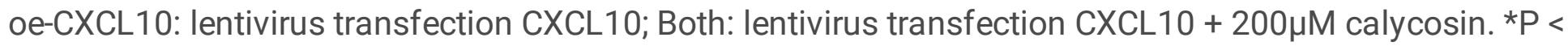
0.05 vs control. \#P<0.05, compared with either CA treatment or CXCL10 transfection alone.
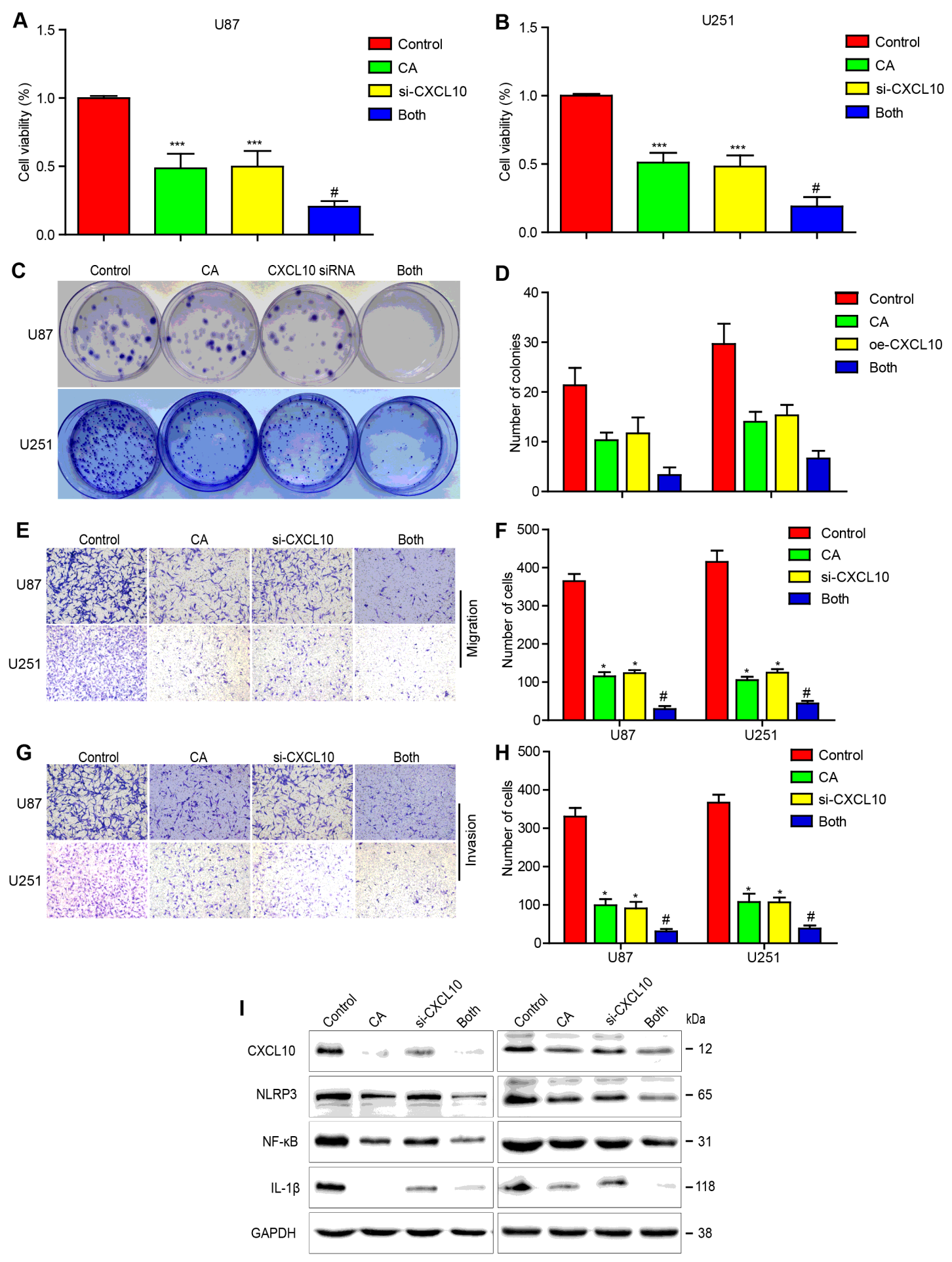

Figure 4 
Downregulating CXCL10 enhances the impact of calycosin on the proliferative, migratory and invasive abilities of GBM cells. (A-D) Effects of CXCL10 knockdown on calycosin in U87 and U251 cell proliferation. (E-H) Effects of downregulating CXCL10 on CA-treated U87 and U251 cell migration and invasion. (I) Downregulating CXCL10 promotes CA-induced CXCL10, NLRP3, NF-KB and IL-1 $\beta$ downregulation. Control: siRNA negative control transfection; CA: siRNA negative control transfection +

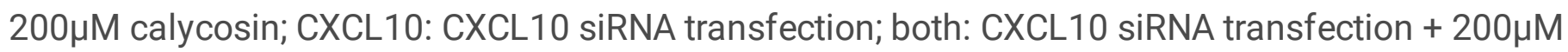
calycosin. ${ }^{*} \mathrm{P}<0.05$ vs control. \#P $<0.05$, compared with either CA treatment or CXCL10 siRNA alone.
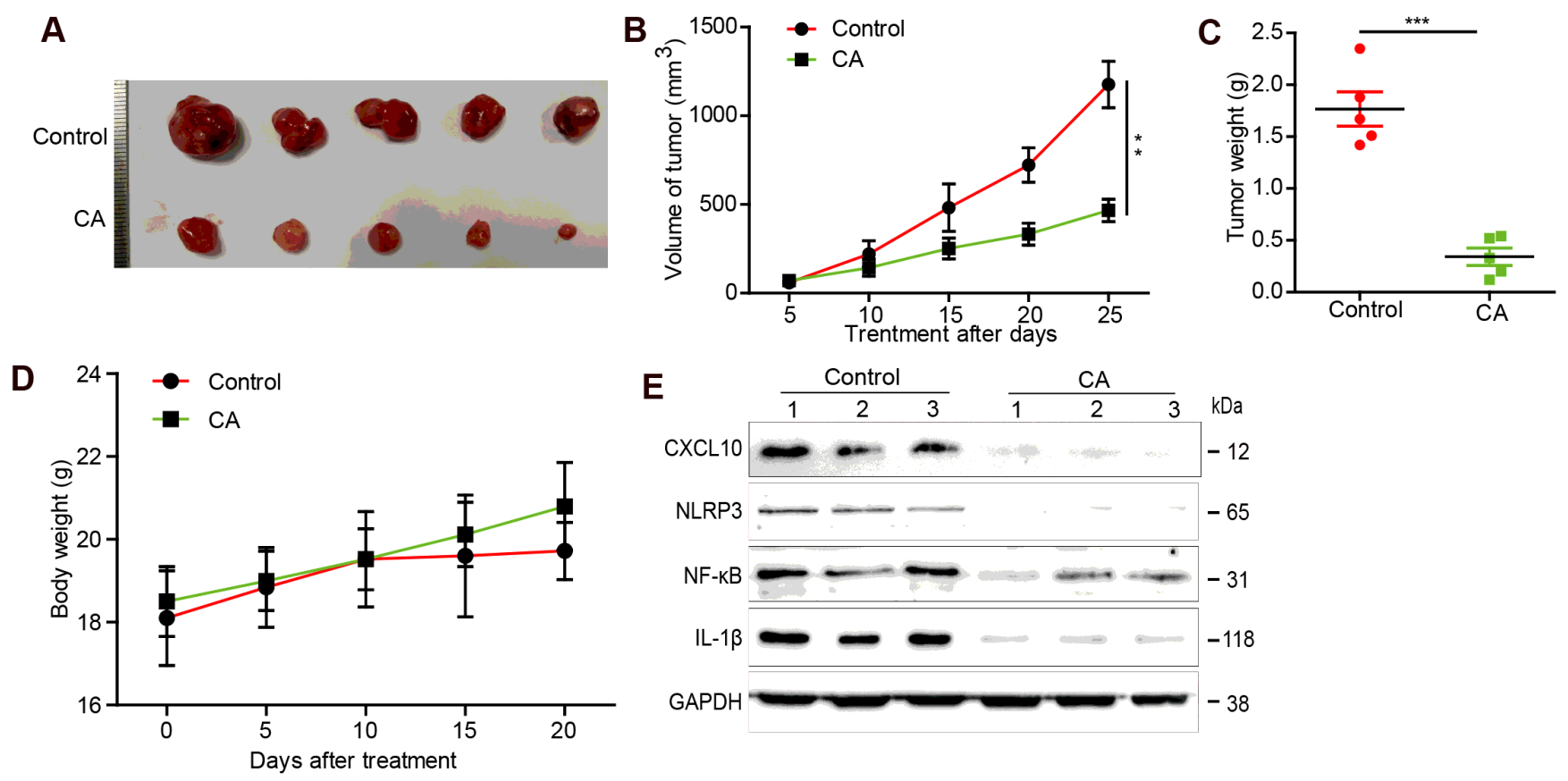

\section{Figure 5}

Effects of calycosin in xenograft mouse models. (A) Xenograft U87 tumors were monitored for size. (B) Mice body weights and tumor volumes were assessed every 5 days. (C) Tumors were harvested and mice were weighed at the end of the experimental period (D) Mice body weights were assessed at the indicated time point. (E) Protein lysates derived from harvested tumor tissues were evaluated for various protein expressions ${ }^{*} \mathrm{P}<0.05$, compared with control. 